\title{
A Simple Method to Determine the Performance of Two-Coil Wireless Power Transfer Systems without Direct Output Measurement
}

\author{
Lucas Ricken Garcia and Paulo J. Abatti
}

\begin{abstract}
Two-coil wireless power transfer (WPT) systems are composed of two circuits tuned at the same resonance frequency, one containing the source, and other containing the load, both connected to each other by the mutual inductance. The power delivered to the load circuit $\left(P_{O}\right)$ divided by the total power supplied by the source $\left(P_{T}\right)$ and by the maximum ideal amount of power which can be delivered to the load circuit are usual figures of merit known as efficiency $(\eta)$ and power transfer capability $\left(P^{*}\right)$, respectively. Additionally, it can be defined a third figure of merit $\left(I^{*}\right)$ as the power dissipated at the source circuit divided by $P_{T}$. It has been recently demonstrated that $\boldsymbol{\eta}$ and $P^{*}$ are related to $I^{*}$. In this paper, it is presented a simple method to monitor $I^{*}$, allowing consequently the determination of $\eta$ and/or $P^{*}$ without any direct measurement at the load circuit. The qualities and limitations of the proposed method are discussed in details. Practical results are included to verify the proposal.
\end{abstract}

Index Terms- Wireless power transfer, inductive power transfer, figures of merit, efficiency, power transfer capability.

\section{INTRODUCTION}

$\mathrm{W}$ IRELESS power transfer (WPT) systems via inductive coupling was first reported by Tesla more than a century ago [1]. The simplest WPT systems are composed of only two resonant circuits tuned at the same resonance frequency, one containing the source, and other containing the load, which are magnetically connected by the mutual inductance. Due to its safety, flexibility, and reliability, WPT systems have become a key technology in several fields, such as, for instance, power charging electric vehicles and implantable medical devices [2]-[7].

Manuscript received Month xx, 2xxx; revised Month xx, xxxx; accepted Month $\mathrm{x}, \mathrm{xxxx}$. This work was supported in part by Brazilian Council for Scientific and Technological Development (CNPq) and Brazilian Council for the Improvement of Higher Education (CAPES).

L. Ricken Garcia is with Graduate Program in Electrical Engineering and Industrial Informatics (CPGEI), Federal University of Technology Paraná (UTFPR), 80230-901, Curitiba, Paraná, Brazil. (e-mail: lukas.ricken@gmail.com).

P. J. Abatti is with Graduate Program in Electrical Engineering and Industrial Informatics (CPGEI), Federal University of Technology Paraná (UTFPR), 80230-901, Curitiba, Paraná, Brazil. (e-mail: abatti@utfpr.edu.br).
Perhaps because of their increasing importance, a lot of research efforts have been devoted to the improvement of the overall system efficiency and/or power transfer capability [7]-[13]. The WPT system's efficiency $(\eta)$ can be defined as the power delivered to the load circuit $\left(P_{O}\right)$ divided by the total power supplied by the source $\left(P_{T}\right)$, whereas its power transfer capability $\left(P^{*}\right)$ can be defined as $P_{O}$ divided the maximum ideal amount of power which can be delivered to the load circuit [11].

Independently of the figure of merit used to characterize the WPT system, in some applications the relative position of coils is not fixed, which means that the mutual inductance between them may have a different value each time. In the same way, load characteristics may be dependent of the employed remote device or may vary with time. Implantable medical devices and electric vehicles, which employ WPT systems, are obvious instances of such applications [14]-[17]. Thus, as $\eta$ and $P^{*}$ depend on mutual inductance and/or load value, feedback strategies to allow the WPT system's control have been devised. For example, in [18] and [19] wireless communication scheme had been used to monitor selected parameters at the load circuit and send the information back to the source circuit for control purposes. Unfortunately, classical feedback procedures require additional communication circuits between the source and load ones, increasing consequently the system complexity, volume, and cost [20]. Alternatively, techniques to allow the control and optimization of WPT systems using exclusively information obtained at the source circuit had been proposed [20]-[25]. For instance, if the current in the source circuit depends on the load value, it can be monitored, and this information used to compensate any possible variation on $\eta$ and $P^{*}$ caused by load value modification. However, if the current in the source circuit depends on two elements of the WPT system, such as load and mutual inductance values, it is necessary to monitor at the source circuit at least two independent parameters (equivalent to describe the WPT system from the source circuit using two independent equations). This can be done, for instance, relating load and mutual inductance values to the real and imaginary parts of the reflected impedance [20]-[25]. In this way, provided all other parameters are known, the circuit is completely determined, so that the WPT system's $\eta$ and/or $P^{*}$ can be promptly calculated, and control procedures 
implemented.

However, the WPT systems must present minimum losses, so a basic guideline used in their implementation is to minimize the reactive effects. Of course, such a WPT system does not present imaginary part of reflected impedance at the source circuit; therefore, the just described technique (see [20]-[25]) is not anymore applicable. Thus, to preserve the advantages of minimum losses with the possibility of output control using only information obtained at the source circuit a novel approach is necessary.

It has been recently demonstrated that using a third figure of merit $\left(I^{*}\right)$, which can be defined as the power dissipated at the source circuit divided by $P_{T}, P^{*}=4 \eta I^{*}$ and that for two-coil WPT systems $\eta=\left(1-I^{*}\right)$, opening up the possibility to determine $\eta$ and/or $P^{*}$ without any direct measurement at the load circuit [26].

The aim of this paper is to present a simple method to monitor $I^{*}$ of a typical two-coil WPT system with both source and load as series circuits, allowing therefore the determination of $\eta$ and $P^{*}$ using only information obtained at the source circuit. The qualities and limitations of the proposed method are discussed in details. Practical results are included to validate the presented theory.

\section{THEORY}

Fig. 1 shows the schematic view of a typical two-coil WPT system with both source and load as series circuits.

As aforementioned, possible losses due to reactive effects should be reduced. Thus, it is usual to consider all circuits tuned at the same angular frequency $\left(\omega^{-1}=\sqrt{L_{1} C_{1}}=\right.$ $\sqrt{L_{2} C_{2}}$ ), because under this condition the voltages and currents in each circuit are in phase.

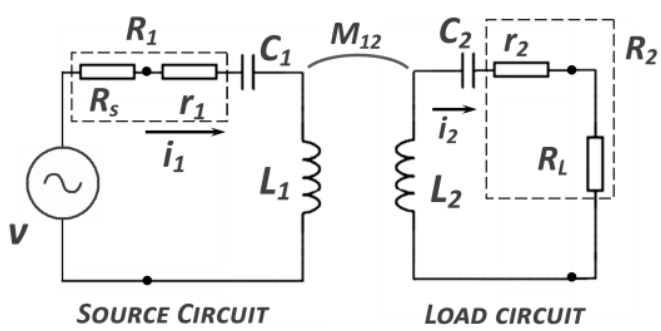

Fig. 1. Schematic diagram of a typical two-coil WPT system with both source and load as series circuits.

Therefore, the two-coil WPT system in practice is composed by two individual circuits with their inductances $\left(L_{1}\right.$ and $\left.L_{2}\right)$ tuned by their respective capacitances $\left(C_{1}\right.$ and $\left.C_{2}\right)$, magnetically connected through the mutual inductance $M_{12}$. The losses at each circuit are represented by the resistances $R_{1}$ and $R_{2}$, which are the sum of the internal resistance of $C_{1}-L_{1}\left(r_{1}\right)$ and the internal resistance of the source $\left(R_{S}\right)$, and the sum of the internal resistance of $C_{2}-L_{2}$ $\left(r_{2}\right)$ and the load resistance $\left(R_{L}\right)$, respectively.

These considerations allow to write

$$
i_{1}=\frac{v}{R_{1}} \frac{1}{\left(1+Q_{12}\right)^{\prime}}
$$

where $Q_{12}$ is defined as

$$
Q_{12}=\frac{\omega^{2} M_{12}^{2}}{R_{1} R_{2}}
$$

Clearly, if $M_{12}=0$, i.e. $Q_{12}=0, i_{1}$ is maximum $\left(i_{1_{M A X}}\right)$, which is given by

$$
i_{1 M A X}=\frac{v}{R_{1}} .
$$

The power dissipated at the source $\left(P_{1}\right)$ and load $\left(P_{O}\right)$ circuits can be written as

and

$$
P_{1}=\frac{v^{2}}{R_{1}} \frac{1}{\left(1+Q_{12}\right)^{2}}
$$

$$
P_{o}=\frac{v^{2}}{R_{1}} \frac{Q_{12}}{\left(1+Q_{12}\right)^{2}} \text {. }
$$

The optimal value of $Q_{12}$ that maximizes $P_{O}$ can be obtained solving $d P_{o} / d Q_{12}=0$, yielding $Q_{12}=1$. Therefore, using $Q_{12}=1$ in (5) the maximum power which can be delivered to the load circuit is $P_{O_{M A X}}=v^{2} / 4 R_{1}$.

The total power delivered by the source $\left(P_{T}\right)$ can be calculated using (4) and (5) yielding

$$
P_{T}=v i_{1}=P_{1}+P_{o}=\frac{v^{2}}{R_{1}} \frac{1}{1+Q_{12}} .
$$

The efficiency $(\eta)$ and the power transfer capability $\left(P^{*}\right)$ can be given, using (4), (5), (6) and $P_{O_{M A X}}$, by

$$
\eta=\frac{P_{o}}{P_{T}}=\frac{Q_{12}}{1+Q_{12}}
$$

and

$$
P^{*}=\frac{P_{o}}{P_{o_{M A X}}}=\frac{P_{o}}{\left(v^{2} / 4 R_{1}\right)}=4 \frac{Q_{12}}{\left(1+Q_{12}\right)^{2}},
$$

respectively.

Observe that dividing (6) by $P_{T}$ gives

$$
1=\frac{P_{1}}{P_{T}}+\frac{P_{o}}{P_{T}} .
$$

The relation $P_{1} / P_{T}\left(=I^{*}\right)$ can be calculated dividing (4) by (6) and using (3) yielding

$$
I^{*}=\frac{P_{1}}{P_{T}}=\frac{1}{1+Q_{12}}=\frac{R_{1} i_{1}^{2}}{v i_{1}}=\frac{i_{1}}{\left(v / R_{1}\right)}=\frac{i_{1}}{i_{1_{M A X}}} .
$$

Finally, using (7) and (10) into (9) and (8) it can be written

$$
\eta=1-I^{*}
$$

and

$$
\mathrm{P}^{*}=4 \eta I^{*}=4\left(1-I^{*}\right) I^{*},
$$

respectively.

Equations (11) and (12) show that $\eta$ and $P^{*}$ can be determined using only information obtained at the source circuit, i.e. monitoring $I^{*}$. In addition, (10) shows that if $i_{1_{\text {MAX }}}$ 
is known, $I^{*}$ can be experimentally determined measuring $i_{1}$.

It was just demonstrated that $P_{O}$ is maximum $\left(P_{O_{M A X}}\right)$ whenever $Q_{12}=1$. Consequently, using (8), the maximum power transfer capability of a two-coil WPT system $\left(P_{M A X}^{*}\right)$ is also equal to 1 . Additionally, using $Q_{12}=1$ into (10) and (11) gives $I^{*}=1 / 2$ and $\eta=1 / 2$, respectively. Thus, following the maximum power transfer theorem maximum $P_{M A X}^{*}=1$ implies $\eta=1 / 2$ [7], [11], and these values can be easily determined since for them necessarily $I^{*}=1 / 2$, or $i_{1}=$ $i_{1_{\text {MAX }}} / 2$.

Moreover, note that $P_{M A X}^{*}=1, \eta=1 / 2$, and $I^{*}=1 / 2$ values do not depend on the individual values of $M_{12}, R_{L}$, and $R_{1}$ since there are innumerous combinations of $M_{12}-R_{L}-$ $R_{1}$ for which $Q_{12}=1$. In other words, independently of $M_{12}$, $R_{L}$, and $R_{1}$ values, whenever $Q_{12}=1, P_{M A X}^{*}=1, \eta=1 / 2$, and $I^{*}=1 / 2$. In fact, each value of $Q_{12}$ correspond to a unique combination of $P^{*}, \eta$, and $I^{*}$ values, whereas innumerous combinations of $M_{12}-R_{L}-R_{1}$ can result a single $Q_{12}$ value.

There is not an optimal value of $Q_{12}$ that maximizes $\eta$, because it monotonically increases as $Q_{12}$ increases (see (7)). Therefore, $\eta$ can be only determined in a trade-off basis, because to increase it $(\eta>1 / 2)$ it is necessary to decrease the value of $P^{*}$ from its maximum value $\left(P^{*}<1\right)$. For instance, adjusting $Q_{12}=3$ makes $P^{*}=\eta=3 / 4$, and $I^{*}=1 / 4$, or $i_{1}=i_{1_{M A X}} / 4$.

At this point it must be emphasized that $P^{*}$ and $\eta$ are related to the power transferred to the load circuit $\left(r_{2}+R_{L}\right)$ not to the power transferred to load $\left(R_{L}\right)$. As load circuit is a series one, the two-coil WPT system "load power transfer capability" $\left(P_{L}^{*}\right)$ and "load efficiency" $\left(\eta_{L}\right)$ are respectively related to $P^{*}$ and $\eta$ as

and

$$
P_{L}^{*}=\frac{R_{L}}{r_{2}+R_{L}} P^{*}=\frac{1}{1+\frac{r_{2}}{R_{L}}} P^{*}
$$

$$
\eta_{L}=\frac{R_{L}}{r_{2}+R_{L}} \eta=\frac{1}{1+\frac{r_{2}}{R_{L}}} \eta \text {. }
$$

Note that in practice as $r_{2} / R_{L}$ decreases, $P_{L}^{*}$ approaches $P^{*}$ as well as $\eta_{L}$ approaches $\eta$, whereas, $I^{*}$ is not influenced by the relation $r_{2} / R_{L}$. Therefore, multiplying (12) by $R_{L} /\left(r_{2}+\right.$ $R_{L}$ ) and using (13) and (14) it can be written

$$
P_{L}^{*}=4 \eta_{L} I^{*}
$$

Summing up, the performance (represented by $I^{*}, P^{*}$ and/or $\eta$ ) of the two-coil WPT system is determined in a unique form by the $Q_{12}$ value. Moreover, perhaps more important from practical point of view, $P^{*}$ and $\eta$, as well as the relationship between $P_{L}^{*}$ and $\eta_{L}$, can be determined uniquely by $I^{*}$ (see (11) and (12), and (15)), since $Q_{12}$ has a univocal relation to $I^{*}$. On the other hand, innumerous combinations of $M_{12}-R_{L}-R_{1}$ can yield a given value of $Q_{12}$. However, the absolute value of $P_{O}$ is determined, given a voltage source with amplitude " $v$ " and a $Q_{12}$ value, by $R_{1}$ (see (5)) and, from the $P^{*}$ and $\eta$ values, $r_{2} / R_{L}$ determines $P_{L}^{*}$ and/or $\eta_{L}$ (see (13) and (14)), respectively. Although the presented analysis was developed for both source and load as series circuits, evidently it can adapted to most of the other configurations (series-parallel, etc., see for example [23]) calculating the equivalent series circuit.

Clearly, the presented method does allow to easily determine $P^{*}$ and $\eta$ by monitoring $I^{*}$, being recommended whenever the values of $R_{L}$ and/or $M_{12}$ are known or their individual determination are not necessary and/or required.

\section{EXPERIMENTAL VALIDATION}

The experiments were performed using two circular coils with 62 turns of enameled copper wire of $35 \mathrm{AWG}$, both with diameter of $40 \mathrm{~mm}$. The coils have self-inductances of $217 \pm 2.4 \mu H$ with internal resistances of $9.25 \pm 0.25 \Omega$. To monitor the current in the source circuit, a resistance $\left(R_{0}\right)$ of $4.9 \Omega$ (measured at $530 \mathrm{kHz}$ ) was added in series. All these measurements were done using an Agilent 4294A vector impedance analyzer operating at $530 \mathrm{kHz}$.

The source was a Minipa MFG-4201A function generator (internal resistance $R_{S}$ equal to $50 \Omega$ ) and the voltages were measured using a Tektronix 2002B oscilloscope.

Both circuits had been empirically tuned at a resonance frequency of $530 \mathrm{kHz}$ using associations of capacitors $(411 \pm 4.2 \mathrm{pF})$. The resistances of the capacitors at $530 \mathrm{kHz}$ were neglected because they were in order of milliohms.

After tuning, the resistances of each circuit yield $R_{1}=63.9 \Omega$, and as three different values of $R_{L}$ were used, $9.9 \Omega, 47.1 \Omega$ and $98.8 \Omega$, the load circuit resistances $\left(R_{2}=r_{2}+R_{L}\right) \quad$ were $19.4 \Omega, \quad 56.6 \Omega$ and $108.3 \Omega$, respectively. All experiments were performed keeping the source circuit unchanged and modifying only $R_{L}$ in the load circuit. Fig. 2 illustrates the experimental setup of the implemented two-coil WPT system.

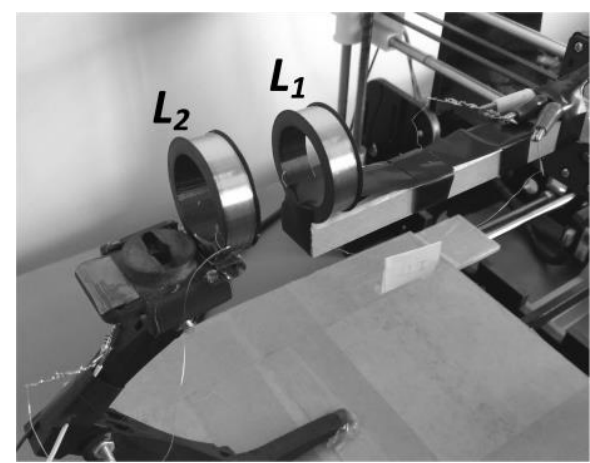

Fig. 2. Experimental setup showing the implemented two-coil WPT system.

In all experiments the mutual inductance $M_{12}$ was gradually varied from $56.5 \mu H$ to $1.4 \mu H$. In a coaxial arrangement these mutual inductances values are related to $L_{1}-L_{2}$ distances (measured between the last turn of $L_{1}$ and first turn of $L_{2}$ ) of $4 \mathrm{~mm}$ and $80 \mathrm{~mm}$, respectively.

Practical data were obtained by measuring the voltages over $R_{0}$ and the load to determine source and load circuit currents, respectively. Theoretical values were calculated using 
Neumann equation to determine the mutual inductance and (7), (8), and (10) to obtain $\eta, P^{*}$, and $I^{*}$, respectively.

The $i_{1_{\text {MAX }}}$ was measured by placing $L_{2}$ sufficiently far away so that $M_{12} \approx 0\left(Q_{12} \approx 0\right)$. The data via $I^{*}$ were obtained dividing $i_{1}$ by $i_{1_{M A X}}$ and applying (11) and (12) to obtain $\eta$ and $P^{*}$, respectively. These values of $\eta$ and $P^{*}$ and those of $r_{2} / R_{L}$ according to the selected $R_{L}$ were used in (13) and (14) to compute $\eta_{L}$ and $P_{L}^{*}$, respectively.

Fig. 3 presents the theoretical (lines) and experimental (points) results of $P^{*}, \eta$, and $I^{*}$ as function of $Q_{12}$, for $R_{1}=63.9 \Omega$, and $R_{2}$ equal to $19.4 \Omega, 56.6 \Omega$ and $108.3 \Omega$.

The practical, both via direct load circuit measurements and using only measured values of $I^{*}$ in (11) and (12), and theoretical results of $I^{*}, P^{*}$, and $\eta$ as function of $M_{12}$ are presented in Fig. 4, for $R_{1}=63.9 \Omega$, and $R_{2}$ equal to $19.4 \Omega$, $56.6 \Omega$ and $108.3 \Omega$.

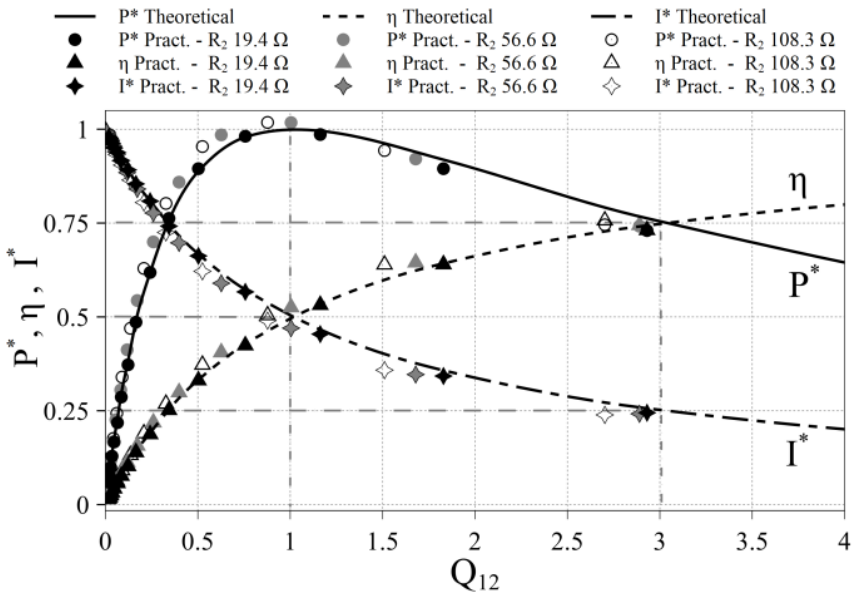

Fig. 3. Theoretical and practical results of $P^{*}, \eta$, and $I^{*}$ as function of $Q_{12}$, for $R_{1}=63.9 \Omega$, and $R_{2}$ equal to $19.4 \Omega, 56.6 \Omega$ and $108.3 \Omega$. The points for $Q_{12}$ equal to 1 and 3 are indicated by vertical dashed lines, whereas horizontal dashed lines indicate the correspondent values of $P^{*}, \eta$, and/or $I^{*}$.

Clearly, the only difference between Fig. 3 and 4 is that, as $I^{*}, P^{*}$, and $\eta$ are plotted as function of $M_{12}$, the results are split into three curves, one for each $R_{2}$ selected value. Fig. 4 shows also that as $R_{2}$ increases a higher value of $M_{12}$ is necessary for $P^{*}$ to reach its maximum value. For example, for $R_{2}$ equal to $19.4 \Omega$ and $56.6 \Omega$ the implemented two-coil WPT system reaches $P_{M A X}^{*}$ for $M_{12} \approx 11.4 \mu H$ and $M_{12} \approx$ $18.1 \mu \mathrm{H}$, respectively.

The influence of $R_{L}$ on the results can be analyzed plotting $P_{L}^{*} \quad$ and/or $\eta_{L}$ in Fig. 3 and/or 4. However, as $I^{*}$ is not influenced by $r_{2} / R_{L}$, perhaps the most appropriate manner to discuss the qualities and limitations of the proposed method is using a graph $I^{*}$ versus $P_{L}^{*}$ and $\eta_{L} \quad$ (Fig. 5), where for comparison purposes, $P^{*}$ and $\eta$ are also presented. In this figure, it had been used the values of $r_{2} / R_{L}$ of approximately $0.96,0.20$, and 0.10 , corresponding to $r_{2}=9.5 \Omega$, and $R_{L}$ equal to $9.9 \Omega, 47.1 \Omega$ and $98.8 \Omega$, respectively, whereas $P^{*}$ and $\eta$ are presented as $r_{2} / R_{L}$ equal to zero.
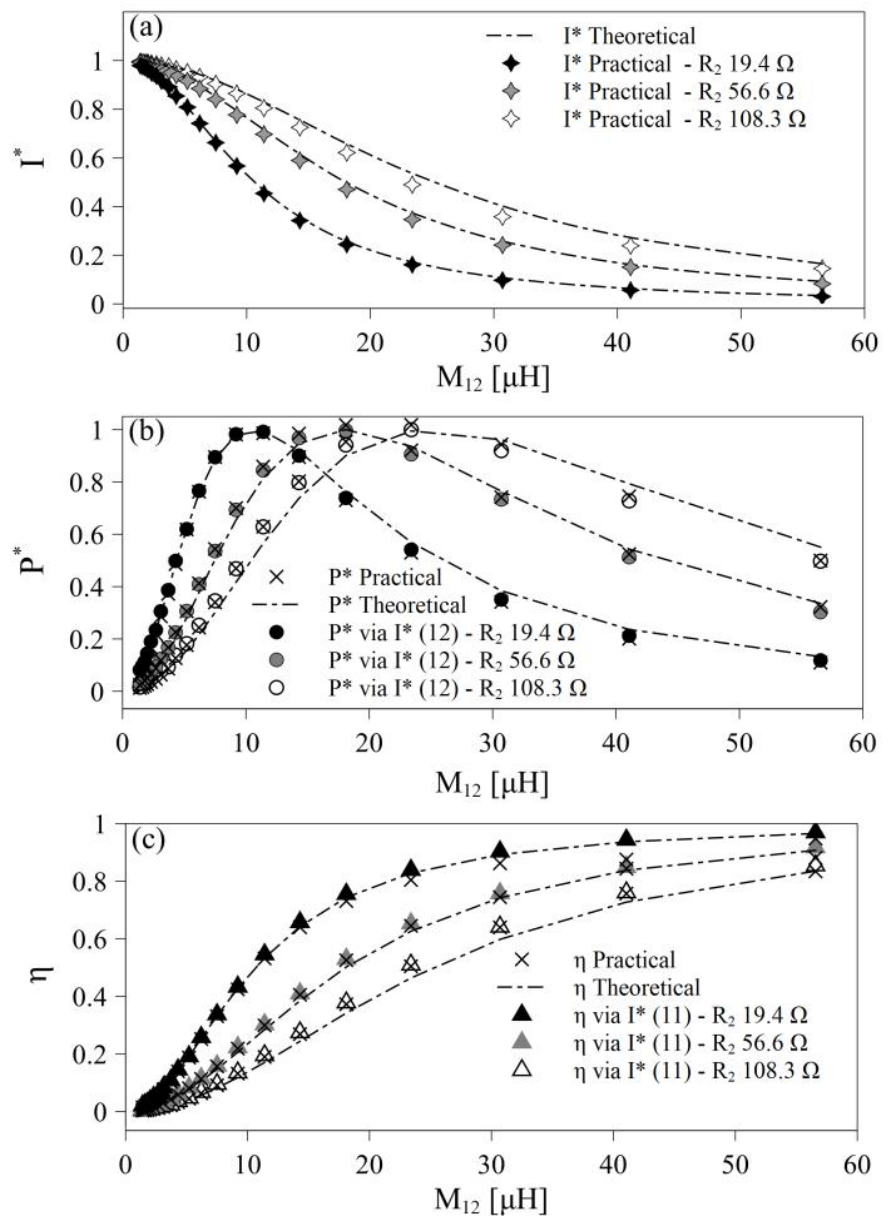

Fig. 4. Practical using direct output measurements and via $I^{*}$ and theoretical results for the two-coil WPT system's (a) $I^{*}$, (b) $P^{*}$ and (c) $\eta$ for $R_{1}=63.9 \Omega$, and $R_{2}$ equal to $19.4 \Omega, 56.6 \Omega$ and $108.3 \Omega$..

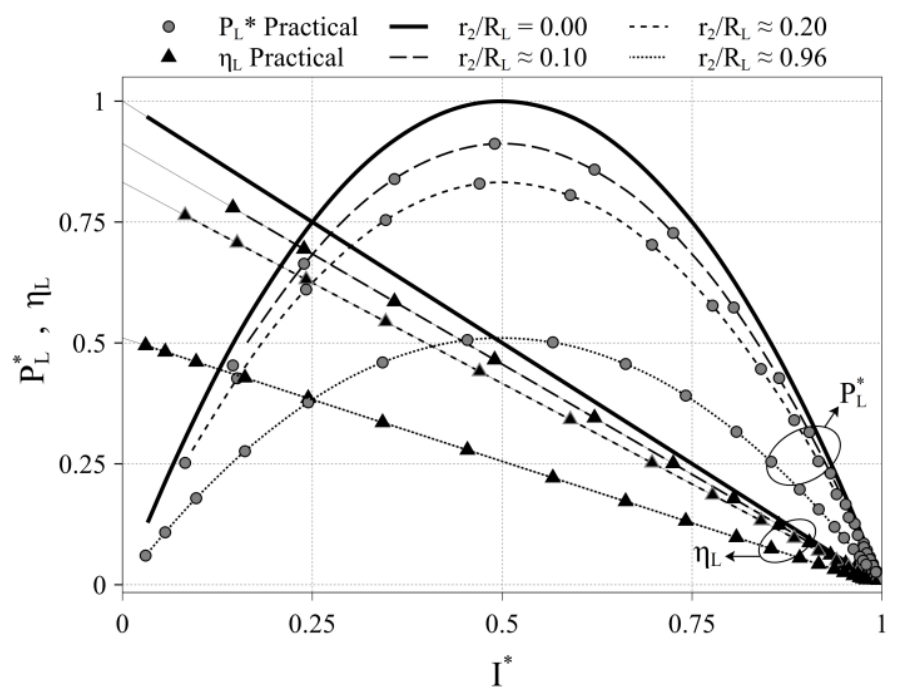

Fig. 5. Behavior of $P_{L}^{*}$ and $\eta_{L}$ relative to $I^{*}$ for different $r_{2} / R_{L}$ : approximately $0.96,0.20$, and 0.10 , corresponding to $r_{2}=9.5 \Omega$, and $R_{L}$ equal to $9.9 \Omega$, $47.1 \Omega$ and $98.8 \Omega$. For comparison purposes, $P^{*}$, and $\eta$ are also presented as $r_{2} / R_{L}$ equal to zero.

This figure summarizes the objective of the present work. For instance, it can be easily observed that if $I^{*}=0.25, P_{L}^{*}=$ $\eta_{L}$ equal to $0.38,0.62$ and 0.68 for $r_{2} / R_{L}$ equal to $0.96,0.2$ 
and 0.1 , respectively, i.e. as $r_{2} / R_{L}$ decreases $P_{L}^{*}=\eta_{L}$ approaches $P^{*}=\eta$, in this case 0.75 . Moreover, if one want to operate the WPT system with an efficiency $\eta_{L}$ higher than $0.75(75 \%)$ and knows that the maximum value of $r_{2} / R_{L}$ in this system is 0.1 , then he or she may modify the relative position of the coils modifying $M_{12}$, so that $I^{*}$ is adjusted to be always smaller than 0.175 .

Finally, it is possible to verify that $P^{*}$ and $\eta$, as well as $P_{L}^{*}$ and $\eta_{L}$, present, for all values of $R_{2}, r_{2}$, and $R_{L}$ utilized, a correlation higher than 0.996 between theoretical and experimental (both direct load circuit measurement and determined via $I^{*}$ ) data.

\section{CONCLUSION}

In this paper it was presented a simple method to monitor $I^{*}$ of a typical two-coil WPT system with both source and load as series circuits, allowing therefore the determination of $\eta$ and $P^{*}$ using only information obtained at the source circuit.

In fact, it was demonstrated that typical two-coil WPT systems have unique set of $I^{*}, P^{*}$ and $\eta$ as function of $Q_{12}$, and as the relation between $Q_{12}$ and $I^{*}$ is univocal, determining $I^{*}$, means that both $P^{*}$ and $\eta$ can be determined. It was also shown that the influence of $R_{L}$ can be calculated comparing it with $r_{2}$ so that the performance of practical WPT systems can be conveniently represented by $P_{L}^{*}$ and/or $\eta_{L}$ (see (13) and (14)). The diagram $I^{*}$ versus $P_{L}^{*}$ and $\eta_{L}$ illustrates the proposed method (see Fig. 5). It must be emphasized however that innumerous combinations of $M_{12}-R_{L}-R_{1}$ can yield a given value of $Q_{12}$ so indirectly $I^{*}$. This means that $P_{L}^{*}$ and $\eta_{L}$ are not determined by the individual values of $M_{12}, R_{L}$ and $R_{1}$ (see (15)); the individual influence of $R_{1}$ is mainly on the absolute value of $P_{O}$ (see (5)), whereas, as just mentioned, $R_{L}$, compared with $r_{2}$, allows to compute $P_{L}^{*}$ and $\eta_{L}$ from $P^{*}$ and $\eta$ values, respectively.

It must be emphasized that, after a simple procedure to determine the amplitude of $i_{1_{M A X}}, I^{*}$ can be promptly determined monitoring the amplitude of $i_{1}$, facilitating any scheme of $P^{*}$ and/or $\eta$ (or even of $P_{L}^{*}$ and/or $\eta_{L}$ if $r_{2} / R_{L}$ is known) control. Moreover, as most of two-coil WPT system configurations can be converted into source and load series circuits, the developed analysis can be adapted to most of the practical circuit.

The authors hope that the presented work may be useful to those interested in design and implementation of WPT systems.

\section{REFERENCES}

[1] N. Tesla, "Apparatus for transmitting electrical energy," U.S. Patent 1119732, 01-Dec-1914.

[2] A. K. Ramrakhyani and G. Lazzi, "On the design of efficient multi-coil telemetry system for biomedical implants," IEEE Trans. Biomed. Circuits Syst., vol. 7, no. 1, pp. 11-23, 2013.

[3] T. Campi, S. Cruciani, F. Palandrani, V. De Santis, A. Hirata, and M. Feliziani, "Wireless power transfer charging system for AIMDs and pacemakers," IEEE Trans. Microw. Theory Tech., vol. 64, no. 2, pp. 633-642, 2016.
[4] X. Li, Y. P. Li, C. Y. Tsui, and W. H. Ki, "Wireless Power Transfer System with \$ISigmalDelta\$-Modulated Transmission Power and Fast Load Response for Implantable Medical Devices," IEEE Trans. Circuits Syst. II Express Briefs, vol. 64, no. 3, pp. 279-283, 2017.

[5] S. I. Ruddell, U. K. Madawala, and D. J. Thrimawithana, "A Wireless EV Charging Topology with Integrated Energy Storage," IEEE Trans. Power Electron., vol. 35, no. 9, pp. 8965-8972, Jan. 2020.

[6] Z. Zhang, H. Pang, A. Georgiadis, and C. Cecati, "Wireless Power Transfer - An Overview," IEEE Trans. Ind. Electron., vol. 66, no. 2, pp. 1044-1058, 2019.

[7] S. Y. R. Hui, W. Zhong, and C. K. Lee, "A critical review of recent progress in mid-range wireless power transfer," IEEE Trans. Power Electron., vol. 29, no. 9, pp. 4500-4511, 2014.

[8] U. M. Jow and M. Ghovanloo, "Design and optimization of printed spiral coils for efficient inductive power transmission," Proc. IEEE Int. Conf. Electron. Circuits, Syst., vol. 1, no. 3, pp. 70-73, 2007.

[9] K. Huang, Y. Zhou, X. Wu, W. Liu, and Z. Yang, "Design and Optimization of Inductive Power Link for Biomedical Applications," Appl. Biomed. Eng., 2011.

[10] M. Kiani, S. Member, M. Ghovanloo, and S. Member, "A Figure-of-Merit for Designing High-Performance Inductive Power Transmission Links," IEEE Trans. Ind. Electron., vol. 60, no. 11, pp. 5292-5305, 2013.

[11] P. J. Abatti, S. F. Pichorim, and C. M. de Miranda, "Maximum power transfer versus efficiency in mid-range wireless power transfer systems," J. Microwaves, Optoelectron. Electromagn. Appl., vol. 14, no. 1, pp. 97-109, Jun. 2015.

[12] Z. Miao, D. Liu, and C. Gong, "Efficiency Enhancement for an Inductive Wireless Power Transfer System by Optimizing the Impedance Matching Networks," IEEE Trans. Biomed. Circuits Syst., vol. 11, no. 5, pp. 1160-1170, 2017.

[13] W. Hao, L. Zhang, S. Xiu, and C. Yang, "Load Segment Tracking Control of IPT System Based on Power Efficiency Optimization," IEEE Access, vol. 8, pp. 7070-7080, 2020.

[14] Z. Bi, T. Kan, C. C. Mi, Y. Zhang, Z. Zhao, and G. A Keoleian, "A review of wireless power transfer for electric vehicles: Prospects to enhance sustainable mobility," Appl. Energy, vol. 179, pp. 413-425, 2016.

[15] N. A. Khan, S. Member, H. Matsumoto, and O. Trescases, "Wireless Electric Vehicle Charger with Electromagnetic Coil Based Position Correction using Impedance and Resonant Frequency Detection," IEEE Trans. Power Electron., vol. 35, no. 8, pp. 1-1, 2020.

[16] A. Yakovlev, S. Kim, and A. Poon, "Implantable biomedical devices: Wireless powering and communication," IEEE Commun. Mag., vol. 50, no. 4, pp. 152-159, 2012.

[17] Y. Zou, S. Member, and S. O. Driscoll, "Implant Position Estimation Via Wireless Power Link," IEEE Trans. Circuits Syst., vol. 62, no. 2, pp. 139-143, 2015.

[18] P. Si, A. P. Hu, S. Malpas, and D. Budgett, "A frequency control method for regulating wireless power to implantable devices," IEEE Trans. Biomed. Circuits Syst., vol. 2, no. 1, pp. 22-29, 2008.

[19] H. L. Li, A. P. Hu, G. A. Covic, and C. Sen Tang, "A new primary power regulation method for contactless power transfer," Proc. IEEE Int. Conf. Ind. Technol., pp. 1-5, 2009.

[20] U. K. Madawala and D. J. Thrimawithana, "New technique for inductive power transfer using a single controller," IET Power Electron., vol. 5, no. 2, pp. 248-256, 2012.

[21] J. P. W. Chow and H. S. H. Chung, "Use of primary-side information to perform online estimation of the secondary-side information and mutual inductance in wireless inductive link," Conf. Proc. - IEEE Appl. Power Electron. Conf. Expo. - APEC, vol. 2015-May, no. May, pp. 2648-2655, 2015.

[22] L. Sun, M. Sun, D. Ma, and H. Tang, "Detecting load resistance and mutual inductance in series-parallel compensated wireless power transfer system based on inputside measurement," Int. J. Antennas Propag., vol. 2018, 2018.

[23] Y. G. Su, L. Chen, X. Y. Wu, A. P. Hu, C. Sen Tang, and X. Dai, "Load and Mutual Inductance Identification from the Primary Side of Inductive Power Transfer System with 
Parallel-Tuned Secondary Power Pickup," IEEE Trans. Power Electron., vol. 33, no. 11, pp. 9952-9962, 2018.

[24] J. Yin, D. Lin, C. K. Lee, and S. Y. R. Hui, "A systematic approach for load monitoring and power control in wireless power transfer systems without any direct output measurement," IEEE Trans. Power Electron., vol. 30, no. 3, pp. 1657-1667, 2015.

[25] J. Yin, D. Lin, T. Parisini, and S. Y. Ron Hui, "Front-End Monitoring of the Mutual Inductance and Load Resistance in a Series-Series Compensated Wireless Power Transfer System," IEEE Trans. Power Electron., vol. 31, no. 10, pp. 7339-7352, 2016.

[26] L. Ricken Garcia and P. J. Abatti, "The Relationship between some Figures of Merit of a typical N-coil Wireless Power Transfer System." unpublished.

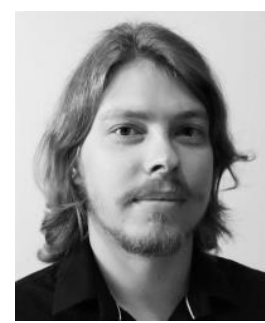

Lucas Ricken Garcia was born in Paranavaí, Paraná, Brasil. He received the B.Eng. degree in Electronic and M.S. degree in Electrical Engineering and Industrial Informatics from Federal University of Technology - Paraná (UTFPR), Brazil, in 2014 and 2016, respectively, where he is currently working toward the D.S. degree in Electrical Engineering and Industrial Informatics.

Since 2016, he has been with Federal University of Technology - Paraná, campus Campo Mourão, Brazil, where since 2018, he has been an Associated Professor with the Department of Electronic Engineering. His research interests include wireless power transfer and instrumentation.

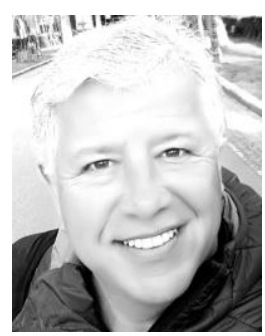

Paulo José Abatti was born in Curitiba, Paraná, Brazil. He received the Bachelor degree in Electrical Engineering from Federal University of Paraná (UFPR), Brazil, in 1980, Master degree in Electrical Engineering from Campinas State University (UNICAMP), Brazil, in 1983, and Doctor degree in Electrical Engineering from Tokyo Institute of Technology (TIT), Japan, in 1991.

Since 1977, he has been with Federal University of Technology - Paraná (UTFPR), Curitiba, Brazil where he is currently titular professor. His research interests include development of sensors, biomedical instrumentation and wireless power transfer techniques. 